\title{
Lubrication forces in air and accommodation coefficient measured by a thermal damping method using an atomic force microscope
}

\author{
Christopher D. F. Honig, ${ }^{1,2}$ John E. Sader, ${ }^{3}$ Paul Mulvaney, ${ }^{4}$ and William A. Ducker ${ }^{1,2, *}$ \\ ${ }^{1}$ Department of Chemical Engineering, Virginia Tech, Blacksburg 24061, USA \\ ${ }^{2}$ Department of Chemical and Biomolecular Engineering, University of Melbourne, Melbourne 3010, Australia \\ ${ }^{3}$ Department of Mathematics and Statistics, University of Melbourne, Melbourne 3010, Australia \\ ${ }^{4}$ Bio21 Institute and School of Chemistry, University of Melbourne, Melbourne 3010, Australia
}

(Received 4 December 2009; published 6 May 2010)

\begin{abstract}
By analysis of the thermally driven oscillation of an atomic force microscope (AFM) cantilever, we have measured both the damping and static forces acting on a sphere near a flat plate immersed in gas. By varying the proximity of the sphere to the plate, we can continuously vary the Knudsen number (Kn) at constant pressure, thereby accessing the slip flow, transition, and molecular regimes at a single pressure. We use measurements in the slip-flow regime to determine the combined slip length (on both sphere and plate) and the tangential momentum accommodation coefficient, $\sigma$. For ambient air at $1 \mathrm{~atm}$ between two methylated glass solids, the inverse damping is linear with separation and the combined slip length on both surfaces is $250 \mathrm{~nm} \pm 100 \mathrm{~nm}$, which corresponds to $\sigma=0.77 \pm 0.24$. At small separations $(\mathrm{Kn}>0.4)$ the measured inverse damping is no longer linear with separation, and is observed to exhibit reasonable agreement with the Vinogradova formula.
\end{abstract}

DOI: 10.1103/PhysRevE.81.056305

PACS number(s): 47.10.ad, 47.45.-n, 07.79.Lh, 68.03.-g

\section{INTRODUCTION}

The Navier-Stokes equation together with the no-slip boundary condition describes the flow of fluids (gases or liquids) within the continuum approximation. In a gas, this framework begins to breakdown when the mean free path $\lambda$ can no longer be considered small relative to a characteristic length scale for the flow, $L$. The ratio of these lengths is known as the Knudsen number,

$$
\mathrm{Kn}=\frac{\lambda}{L} .
$$

Low Knudsen number $(\mathrm{Kn} \ll 1)$ implies that a continuum description is appropriate, and the conventional NavierStokes treatment suffices. At higher Knudsen number, however, nonequilibrium effects in the gas become significant, and more sophisticated treatments based on the Boltzmann equation are required. In the limit $\mathrm{Kn} \rightarrow \infty$, the molecules no longer interact with each other, and a free molecular analysis is applicable.

Examples in which high Knudsen number flows can occur include the flow of gas through nanoscale pores or membranes, and within hard disk recording heads. The growing number of applications involving microfluidics and nanoelectromechanical system (NEMS) devices has led to a surge of interest in studying fluid dynamics beyond the conventional Navier-Stokes treatment. Equivalently, if the gas is at low pressure, then high Knudsen number flows occur at macroscopic length scales. For example, high altitude aircraft, satellites, and atmospheric re-entry vehicles all encounter flows with high Knudsen number regimes. It is therefore of

\footnotetext{
*wducker@vt.edu
}

scientific interest to explore the fundamental physics behind such flows.

The flow of gases at small but finite Knudsen number, i.e., in the slip-flow regime, can be calculated using slip models derived from the kinetic theory of gases. The slip-flow regime is conventionally considered to lie within the range $0.001 \leqq \mathrm{Kn} \lesssim 0.1$, whereas the transition regime $(0.1 \leqq \mathrm{Kn}$ $\$ 10)$ lies between the slip-flow and free molecular regimes. Slip models are derived from a formal asymptotic expansion of the Boltzmann equation in the limit of small Knudsen number [1]. To leading order, the no-slip boundary condition is recovered, and to next order the so-called first-order slip condition appears with a specified slip length. Vitally the derived slip condition is a leading order asymptotic result and, for this reason, is only applicable in the small Knudsen number regime. It cannot be applied in the free-molecule flow regime, which in our experiments occurs for very small separations.

The tangential velocity for the first-order slip boundary condition derived from the Boltzmann equation with hard spheres is $[2,3]$

$$
v_{t}=\boldsymbol{v} \cdot \hat{\mathbf{t}}=\lambda \xi(\sigma) \hat{\mathbf{n}} \cdot \mathbf{e} \cdot \hat{\mathbf{t}},
$$

where

$$
\xi(\sigma)=\frac{2.01}{\sigma}-0.73-0.16 \sigma,
$$

and $\hat{\mathbf{n}}$ and $\hat{\mathbf{t}}$ are the unit vectors normal and tangential to the surface, respectively, whereas $\boldsymbol{v}$ and $\mathbf{e}$ are the velocity field and rate-of-strain tensor in the gas, respectively.

Equation (2) was obtained using Maxwell's simple boundary condition. The tangential momentum accommodation coefficient, $\sigma$, specifies the proportion of gas molecules that are diffusely reflected from the surface to those that undergo specular reflection. Specifically, $\sigma=1$ corresponds to 
pure diffuse reflection and $\sigma=0$ yields pure specular reflection.

For shear flow past a solid surface whose unit normal vector $\hat{\mathbf{n}}$ is in the $z$ direction, Eq. (2a) becomes

$$
\left.v\right|_{z=0}=\left.\lambda \xi(\sigma) \frac{\partial v}{\partial z}\right|_{z=0},
$$

where $v$ is the component of the velocity tangential to the surface.

Critically, this is a "macroscopic" boundary condition for the Navier-Stokes equation and, as such, does not explicitly describe the flow profile within a few mean-free pathlengths from the solid. Therefore, the slip length $\lambda \xi(\sigma)$ predicted by Eq. (3) represents the distance into the solid where the velocity would be zero if the velocity profile was extrapolated from distances greater than a few mean-free pathlengths.

The slip length may vary from $1.12 \lambda$ (for full tangential momentum accommodation) to infinity (no accommodation). In the former case, the incoming gas molecules are reemitted from the wall with a Maxwellian distribution at the wall temperature. In the latter case, the molecules undergo pure reflection with no accommodation. It is important to note that even in the case of pure diffuse reflection $(\sigma=1)$, the slip length is finite and is of the order of the gas mean-free path.

We note that the traditional Navier slip condition, which is simply Eq. (3) but with an arbitrary slip length, is commonly used to quantify "slip" flows in liquids. $[4,5]$ However, there is no theoretical justification for using such a model, and, in any case, recent experiments demonstrate that the no-slip boundary condition is very accurately obeyed in Newtonian liquids [6-8], so there may be little need for such a model.

The experimental determination of accommodation coefficients is usually achieved by (1) measuring the energy of a reflected beam of molecules striking a surface [9] or (2) by confining the gas to a small length scale (such as a microchannel [10] or a spinning rotor gauge [11]). [12,13] In molecular beam experiments, a beam of gas molecules is fired at a fixed angle against a surface with a specified energy and momentum whereas in confined length scale experiments, the incident gas molecules have a random distribution of energy, momentum and angle. Thus beam accommodation coefficients are not directly comparable with our results or other confined length scale experiments.

A problem specific to gas flow measurements at small length scales is that the resulting mass fluxes are very small and prone to significant experimental uncertainty. [10] During confinement, it is also difficult to quantify the geometry of the small devices, which is essential in extracting accurate slip-length measurements. These two problems can be overcome using a colloidal probe atomic force microscope (AFM), which is able to (a) accurately confine a gas to very small length scales (1-10 $000 \mathrm{~nm})$ and (b) accurately measure very small forces that arise from the gas flow, and therefore can be used to test models of the gas flow. It has been used to measure the accommodation coefficient and slip length in air in a mechanically driven mode by Maali and Bhushan [14].

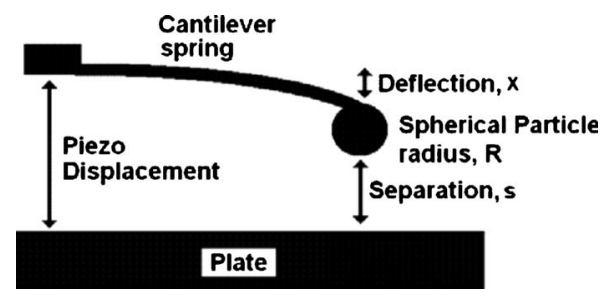

FIG. 1. Schematic of the colloidal probe AFM experiment. A sphere is attached to the end of the cantilever. The cantilever may deflect due to thermal oscillations or static surface forces.

Colloidal probe AFM was developed as a force sensing tool simultaneously by Ducker and co-workers $[15,16]$ and Butt [17] almost two decades ago. Since then it has been repeatedly applied to measure surface forces with a resolution of $10^{-13} \mathrm{~N}$ in force and $10^{-10} \mathrm{~m}$ in separation. The technique uses a cantilever connected to a sample plate via a piezoelectric translation stage (see Fig. 1). A colloidal sphere can be mounted to the free end of the cantilever (a) to increase the magnitude of forces, and (b) to provide a known and simple geometry. In most commercial AFMs the force is obtained from the change in end slope of the cantilever, which is in turn measured by the light lever method [18]. The separation between the sphere and plate is adjusted by a piezoelectric stage. Note that the separation between the sphere and plate is the sum of the piezo displacement and the deflection.

The disadvantage of the AFM colloidal probe method is that surface asperities on either the sphere or plate complicate the analysis. In an ideal experiment, a perfect sphere interacts with a perfectly smooth flat plate. In practice, the detailed geometry of the interaction zone is not known; yet the analysis proceeds as if the idealized geometry occurred, so there is the possibility of introducing errors. In particular, we, and others, usually define the zero of separation, $s$, as the point where a very high-gradient repulsive force is measured, and it is assumed that the two solids are in contact. The data are then compared to theories based on the idealized geometry using the measured $s=0$. An example of an error would be if the existence of a single asperity on one surface led to a steep repulsive surface force without greatly affecting the lubrication force. Such an asperity would lead to a distance offset between the measured force and the theory that is applied and may be interpreted as a slip length. A second disadvantage is that the geometry is a sphere approaching a flat plate, so the flow profile varies radially and there is a nonuniform shear in the flow.

In this paper, we use AFM to measure lubrication forces between hydrophobic solids in air. These forces are interesting in their own right because of applications to fouling of surfaces with particles and particle spraying applications. These forces arise because of the flow of gas in the thin film between the solids and therefore can be used to test theories of gas flow in confined geometry and to measure accommodation coefficients. We measure forces between hydrophobic solids so as to prevent the formation of a water film, which would complicate the analysis. The forces are measured in two ways, from the direct deflection of the cantilever during a fast approach of the sphere to the plate, and also by the 
analysis of the thermal vibration of the cantilever [19]. We obtain the damping as a function of separation between the sphere and the plate and use this to determine both the slip length in the slip-flow regime, and deviations from slip-flow theory within the transition and free molecular regimes. Recently, Maali and Bhushan [14] have addressed the same problem by measuring the driven oscillation of a cantilever [20].

\section{THEORY}

A lubrication force, $F_{\text {lub }}$, in the $z$ direction acting on the sphere as it approaches an infinite flat plate, can be calculated from the Navier-Stokes equation [21], which together with the first-order slip condition in Eq. (3) gives

$$
F_{\text {lub }}=-\frac{6 \pi \eta R^{2}}{s+\delta_{\text {total }}} \frac{d s}{d t},
$$

where $\eta$ is the fluid viscosity, $R$ is the sphere radius, $s$ is the distance from the solid surface to the point of closest approach to the sphere (see Fig. 1), $t$ is time, and $\delta_{\text {total }}$ is the sum of the individual slip lengths, $\lambda \xi(\sigma)$, [Eq. (3)] from the sphere-medium interface and from the plate-medium interface. The minus sign reflects the fact that lubrication force acts in the opposite direction to the velocity.

To obtain the total slip-length $\delta_{\text {total }}$ from Eq. (4), we need to measure the lubrication force and sphere velocity at a range of separations, $s$. We assume the following equation of motion of the sphere,

$$
m \frac{d^{2} s}{d t^{2}}+D(s) \frac{d s}{d t}+k x=F_{\text {noise }}+F_{\text {surface }}(s),
$$

where $m$ is the effective mass, $s$ is the distance between the closest point of the sphere and the plate, $D$ is the damping coefficient, $k$ is the spring constant, $x$ is the deflection of the cantilever from its equilibrium state, $F_{\text {noise }}$ is the noise in the system (assumed to be dominated by thermal noise), and $F_{\text {surface }}$ is the thermodynamic surface force acting between the particle and the plate. The damping coefficient $D$ includes both the lubrication force on sphere [Eq. (4)] and the damping from the cantilever. Because the problem is linear, these contributions are additive and so we obtain the damping on the sphere by subtracting the cantilever damping from the total damping.

In this paper, we describe measurements of the deflection of an AFM spring which allow us to measure the damping. We do this in two separate parameter regimes that we call the $\mathrm{dc}$ method and the thermal noise method.

\section{A. de method}

In the first method, we drive the cantilever and the piezoelectric translation stage at high velocity (up to $1 \mathrm{~mm} \mathrm{~s}^{-1}$ ) toward the surface, and measure the deflection of the cantilever. The inertial term and thermal noise term in Eq. (5) are small for the conditions in which we analyze our data. The thermal noise is not required for the analysis, and because most of the thermal signal occurs at high frequency, it can be averaged (to zero). The surface force is not negligible and must be subtracted from the measured force by performing an initial force curve at slow speed, where there is no hydrodynamic force.

To obtain the lubrication force on the sphere for comparison to Eq. (4), we must first subtract the damping from the cantilever [7]. By experiment, we find that the measured force is approximately constant at a separation of $10 \mu \mathrm{m}$; we take this as the cantilever drag and subtract it from the total force to get the lubrication force on the sphere.

\section{B. Thermal noise method}

A cantilever at a nonzero temperature will have a thermal energy of order $k_{B} T$, where $k_{B}$ is Boltzmann's constant and $T$ is the absolute temperature. The thermal energy of the cantilever will cause it to oscillate, and these oscillations are routinely used to calibrate the cantilever spring constant [22-24]. The thermal motion of an AFM cantilever near a solid in gases has also been studied [19]. In the thermal noise method, the cantilever is dwelled at a fixed position of the piezoelectric drive near the surface for a fixed time (typically $20 \mathrm{~s}$ in our experiments). The fixed drive position corresponds to an approximately fixed separation. A forceseparation curve is measured immediately before and after the dwell, so that the cantilever contacts the substrate and the separation distance at the beginning and end of the dwell can be measured. During the dwell, the spring deflection is measured at sufficiently high frequency so that the thermal vibrations are resolved. This cantilever dwell process is repeated at various sphere-plate separations so that the thermal noise is know as a function of separation.

Alternatively, the thermal noise can be measured while the cantilever is driven continuously toward the surface at low piezo displacement speeds. Although this continuous thermal noise measurement is easier to execute, three disadvantages arise: (a) the cantilever moves continuously while the thermal vibrations are measured, so noise data from a range of separations must be binned together (b) usually these bins are measured over a shorter time than in the dwell method, so the error is larger and (c) the time between contacting the sphere against the plate is longer. The latter introduces an error in the particle-plate separation because the dimensions of the apparatus change due to small fluctuations in temperature over time.

The cantilever has a mean deflection, $x$, due to surface forces, and a time-varying departure from this deflection, $y$, due to thermal noise. The amplitude of this time-varying deflection is sufficiently small that (a) the damping coefficient is constant, and (b) the surface force can be linearized, leading to [25]

$$
m \frac{d^{2} y}{d t^{2}}+D(s) \frac{d y}{d t}+\left(k-\left.\frac{d F_{\text {surface }}}{d z}\right|_{s}\right) y=F_{\text {noise }},
$$

where $m$ is the effective mass of the cantilever, which comprises of a component due to the surrounding gas [26]. The damping force in Eq. (6) (the second term) is the sum of all damping on the cantilever. We are interested in measuring the component that is caused by the lubrication force, which is described by Eq. (4). 
When we analyze Eq. (6) we make the simplifying assumptions that $m, D(s), F_{\text {surface }}(s)$, and $k$ are all frequency independent over the range of frequencies analyzed. This approximation is very good when the resonant frequency is constant, because we measure over a small, constant, frequency range around the resonance peak. In the presence of a strong surface force or heavy damping the resonance peak may shift or become broader so the frequency dependence of parameters such as the damping or mass may become important. Equation (6) is still valid when the frequency varies, but the damping will become more difficult to extract. For our experiments, the resonant frequency is constant at separations above $100 \mathrm{~nm}$ but may become smaller at smaller separations due to the action of surface forces.

In our application of using Eq. (6) to the thermal method, we assume that (a) the effective mass $m$ of the cantilever is independent of the separation $s$, and (b) the damping is sufficiently small that the resonance peaks are sharp; i.e., they exhibit high quality factors. Both are satisfied in gas [27,28], which facilitates implementation of Eq. (6).

Given these assumptions, Eq. (6) is solved to obtain the amplitude of vibration. The energy spectrum density $\left(E_{\mathrm{SD}}\right)$ in the immediate vicinity of the fundamental resonance is [29]

$$
E_{\mathrm{SD}}=\frac{1}{2} k|A|^{2}=\frac{B}{\left(1-\left[\frac{f}{f_{0}}\right]^{2}\right)^{2}+(2 \pi f)^{2}\left(\frac{D(s)}{k_{\text {total }}(s)}\right)^{2}}+C,
$$

where $f$ is the frequency $(\mathrm{Hz})$ and $f_{0}$ is the resonant frequency of the cantilever in the presence of the surface force, $k_{\text {total }}(s)$ is the coefficient of $y$ in Eq. (6) (effective spring constant), and $A$ is the amplitude of the cantilever at a given frequency due to the thermal motion. As the oscillation amplitude is very small, $k_{\text {total }}(s)$ and $D(s)$ are treated as constant, and can be determined by comparing Eq. (7) to the measured $E_{\mathrm{SD}}$. The coefficients $B$ and $C$ are the fitting parameters. Our experiment measures the end slope, rather than the deflection, and the conversion factor is contained in the constant B. Our experiment thus yields $D$, the total damping coefficient. We subtract from this the damping that occurs when the particle is far from the plate, to obtain the damping that arises from the lubrication force. Equation (4) is used to obtain the fitted combined slip length, $\delta_{\text {total }}$, which is equal to the sum of $\lambda \xi(\sigma)$ for each surface (Eq. (3)). The accommodation coefficient is then obtained from $2 \mathrm{~b}$.

\section{EXPERIMENT}

\section{A. Preparation of solids}

The borosilicate glass spheres mounted to the cantilevers were purchased from Duke Scientific and have a nominal radius of $10 \mu \mathrm{m}$. As described above, asperities on the sphere hinder our ability to compare experiment with theory, so each sphere was imaged on a reverse tip grating after being mounted to a cantilever, and any sphere with a surface asperity greater than $10 \mathrm{~nm}$ in the contact region was discarded (this represents $80 \%-90 \%$ of the mounted spheres). The rms roughness of the sphere surface was $0.7 \mathrm{~nm}$ when imaged over $0.01 \mu^{2}$. The flat plates were glass microscope cover slip procured from Fischer Finest, which have a surface roughness of $0.25 \mathrm{~nm} \mathrm{rms}$ and a typical maximum peak-to-valley roughness of $1.5 \mathrm{~nm}$ over a $4 \mu \mathrm{m}^{2}$ image area. The glass slides were cleaned in oxygen plasma for 30 $\mathrm{s}$ at $20 \mathrm{~W}$ power before a $2 \mathrm{~h}$ vapor deposition of trimethylchlorosilane (TMCS). After the silane deposition the water contact angle on the surface was high $\left(\theta_{\text {advancing }}\right.$ $=85^{\circ}, \theta_{\text {receding }}=70^{\circ}$ ), which inhibits formation of a thin water film on the solid. After the TMCS deposition, the slides were ultrasonicated in toluene for $15 \mathrm{~min}$, then rinsed in Millipore water, and then finally rinsed in ethanol. The slides were blown dry with ultrahigh purity nitrogen after each rinsing step. The sphere cantilevers were cleaned by exposure to a UV lamp for $1 \mathrm{~h}$ before the TMCS vapor deposition. After the silanization, they were rinsed in Millipore water, then ethanol.

\section{B. AFM}

All the data presented here were collected on MFP-3D Asylum Research AFMs when the system was open to the atmosphere. The cantilevers used were rectangular or triangular in geometry (gold coated silicon nitride Veeco MSCT) with spring constants ranging from $0.01-0.05 \mathrm{~N} / \mathrm{m}$ or ORC8 cantilevers (Veeco). In order to determine the damping coefficient from the $E_{\mathrm{SD}}$, the cantilever spring constant must be known. The cantilever spring constant was calibrated using the Hutter method for all cantilevers, and using both the Hutter and Sader methods for the rectangular cantilevers. For the Hutter method, the spring constant was measured after the sphere had been mounted to the cantilever [22], For the Sader method, calibration was performed without the sphere attached (for reasons discussed in [30]) and a correction applied for off-end placement of the sphere. An optical microscope at $50 \times$ magnification was used to determine the sphere loading position on the end of the cantilever. With the sphere mounted, the resonant frequencies of the cantilevers are in the range of $4-8 \mathrm{kHz}$. The reasons for selecting these cantilevers are: (1) in our current experimental setup, the maximum sampling rate is $50 \mathrm{kHz}$. The Nyquist frequency is 25 $\mathrm{kHz}$ so we require a cantilever with a resonant frequency below $25 \mathrm{kHz}$; (2) they have a low spring constant, which improves our sensitivity for the dc measurement; (3) the amplitude of the cantilever oscillation increases with a smaller spring constant, which is favorable for greater sensitivity in the thermal method.

\section{C. dc method}

As the viscosity of air is very low (typically $0.02 \mathrm{mPa} \mathrm{s}$ ) we need to use very low cantilever spring constants (approximately $0.01-0.05 \mathrm{~N} / \mathrm{m}$ ) and very high piezo displacement drive speeds (up to $2 \mathrm{~mm} / \mathrm{s}$ ) to measure the lubrication force. In comparison, the typical AFM surface force measurements are performed at $0.001-0.01 \mathrm{~mm} / \mathrm{s}$. To access these high drive speeds in the AFM, we needed to bypass software limits on our AFM.

Conventional AFM force measurements use a triangle wave displacement for the piezoelectric drive, which neces- 


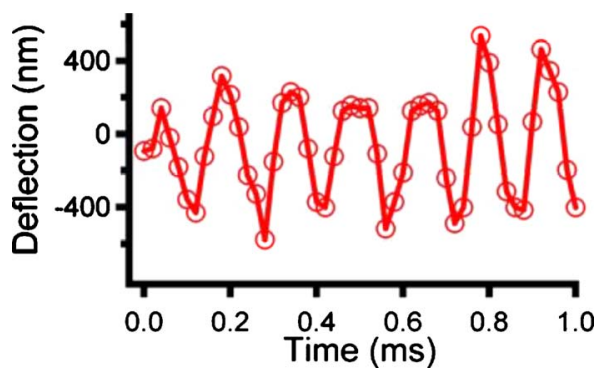

FIG. 2. (Color online) Sample data of time series of deflection during the dwell at a fixed separation. Rectangular silicon nitride, Veeco OCR8 cantilever D, measured spring constant $=0.0548 \mathrm{~N} / \mathrm{m}$, sphere radius $=15.3 \mu \mathrm{m}$

sarily includes high frequency drive components at the start, turn-around and end of the displacement. When operating at high speeds, in a low viscosity medium, this high frequency drive stimulates vibration of the cantilever and the $z$ actuator, which manifest as "ringing" in the end-slope signal. To prevent this ringing, we need to use a waveform without high frequency components. The easiest way for us to do this was to operate the drive with feedback on the LVDT position that contained electronic damping or inertia, i.e., to operate in closed loop rather than open loop configuration. For the MFP-3D, we reduced the integral gain (ZIgain) on the feedback loop from 10.153 (default) to 9.0 to slow down the response time of the feedback loop. To further reduce the ringing of the $z$ stage, we also used a new cantilever holder with a high spring constant.

When operating at high speeds in the AFM, we need to be mindful of filters within the instrument. In Asylum AFMs, there is a filter on the output of the linear variable displacement transducer (LVDT) used to measure the piezo position. To make sure that the LVDT tracked the piezo motion with high precision at high drive speeds, we set this filter [called the Z(LVDT) filter] to $100 \mathrm{kHz}$, its maximum. We also collected data at the maximum collection frequency $(50 \mathrm{kHz})$ and maximized the low pass filter value on this data $(50$ $\mathrm{kHz})$.

\section{Thermal noise method}

The cantilever is dwelled near the surface of the plate for $20 \mathrm{~s}$ and the deflection is recorded at a high data collection frequency of $50 \mathrm{kHz}$ (Fig. 2). The separation distance during the dwell is determined by performing a quick force curve (1 $\mathrm{Hz}$ ) immediately before and after the dwell. Dwells are performed at 20 different separation values so that the thermal motion may be studied as a function of sphere-plate separation. For the purpose of illustration we consider a sample cantilever (Rectangular silicon nitride, Veeco OCR8 cantilever $\mathrm{D}$, measured spring constant $=0.0548 \mathrm{~N} / \mathrm{m}$, sphere radius $=15.3 \mu \mathrm{m})$. The thermal oscillations can be seen on an expanded time axis (Fig. 2). The thermal oscillations from the dwell at each separation are then converted into an energy spectrum density $\left(E_{\mathrm{SD}}\right.$, Fig. 3$)$. This is achieved by dividing each dwell data set into 2000 smaller bins of equal size, performing a fast Fourier transform on each bin, and

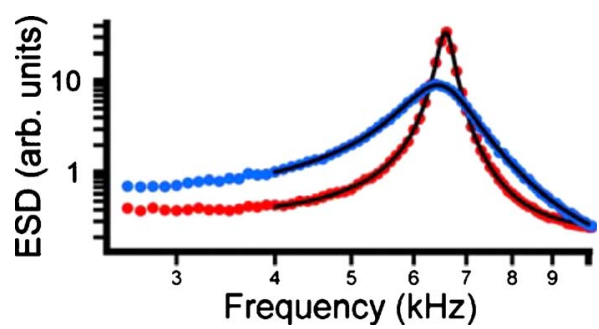

FIG. 3. (Color online) Energy spectrum density of a sample cantilever at $1.091 \mu \mathrm{m}$ separation from the surface (light red, narrower) and $71 \mathrm{~nm}$ separation (dark blue, broader). The black lines show the fit of Eq. (7) to the data. Each $E_{\mathrm{SD}}$ is measured over $20 \mathrm{~s}$. The measured resonant frequency, quality factor and damping are $6.589 \mathrm{kHz}, 19.86,66.1 \mathrm{nN} \mathrm{s} / \mathrm{m}$ (light red, far) and $6.503 \mathrm{kHz}, 4.88$ and $275.5 \mathrm{nn} \mathrm{s} / \mathrm{m}$ (dark blue, close). The same cantilever was used in Figs. 2 and 3.

then summing the square of the magnitude output from each bin in frequency space. Amplitude normalization of the $E_{\mathrm{SD}}$ was not implemented, since only the functional form is required to extract the slip length and viscosity. The output from the fast Fourier transform is the amplitude squared, which is linearly proportional to the energy [Eq. (7)].

When a continuous thermal noise run is performed, the base of the cantilever is driven toward the plate at a low speed (100 s for each for the approach and separation) and the deflection is recorded at a high data collection frequency of $50 \mathrm{kHz}$. The slow approach velocity is necessary because the deflection must be measured over enough time to generate the thermal noise spectrum. If we were to approach at high velocity, during this required time, the sphere would span a range of separations and we would lose separation resolution of the damping and surface forces. The deflection data prior to surface contact is divided into 20 segments of sequential time points. Each segment has exactly the same number of points and is collected over approximately $2 \mathrm{~s}$. Each segment is then converted into an $E_{\mathrm{SD}}$ as above. Because the piezoelectric translation stage continues to move during each segment of deflection data, the $E_{\mathrm{SD}}$ is an average over a small range of separations.

Two sample $E_{\mathrm{SD}}$ plots are shown in Fig. 3. At larger separations, the lubrication force damping on the cantilever is smaller and so the peak around resonance is much sharper [20]. Fits of Eq. (7) to the two $E_{\mathrm{SD}}$ plots are shown as black lines. The high accuracy of the fits to Eq. (7) validates our methodology.

\section{RESULTS}

The force curve-dwell-force curve sequence was performed over a range of separation values from contact to $1 \mu \mathrm{m}$, so the thermal noise method could be implemented. Each dwell period was converted into as $E_{\mathrm{SD}}$ (Fig. 3), as described above. Then from the fit of Eq. (7) to the $E_{\mathrm{SD}}$ at each position, we extracted the damping coefficient (Fig. 4) and resonant frequency (Fig. 5) as a function of separation.

The damping coefficient increases as the separation $s$ gets smaller. This is to be expected from Eq. (4): the lubrication 


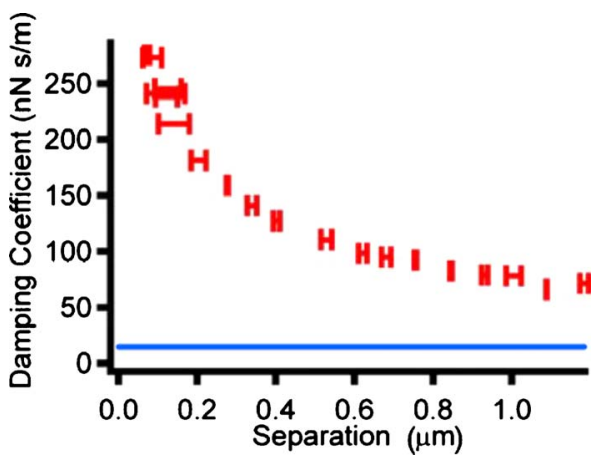

FIG. 4. (Color online) Damping coefficient as a function of separation. The damping coefficient is obtained from the fit of Eq. (7) to the $E_{\mathrm{SD}}$ (Fig. 3) at each separation (red bars). The range of the error bars is the difference between the separation measured by a force curve before and after the dwell. The blue line shows the estimated damping from the cantilever, which we subtract before comparing the data to the theoretical damping on the sphere. The damping from the cantilever alone (with no sphere attached) is shown over a range of separation from $2 R$ to $(2 R+1 \mu \mathrm{m})$ (blue line). The cantilever is the same as used to capture the data in Fig. 2.

force increases with proximity of the sphere to the plate.

As discussed above, the damping force on the cantilever is the sum of two effects: (1) the lubrication force on the sphere (our focus) and (2) the damping force on the cantilever, (which is also potentially a function of separation from the plate). The damping on the cantilever must be subtracted from the total damping to obtain our estimate of the sphere damping on the sphere. To minimize the distancedependence of the cantilever damping, we used narrow rectangular cantilevers (nominal width $20 \mu \mathrm{m}$ ) and large spheres (nominal diameter $30 \mu \mathrm{m}$ ). The important regime for estimating the distance-dependence of the cantilever damping is when the cantilever is separated from the plate by more than $2 R \sim 30 \mu \mathrm{m}$. To examine this regime, we took a cantilever of the same type from the same wafer, but without an attached sphere, and used the thermal noise method to measure the damping as a function of separation. For the

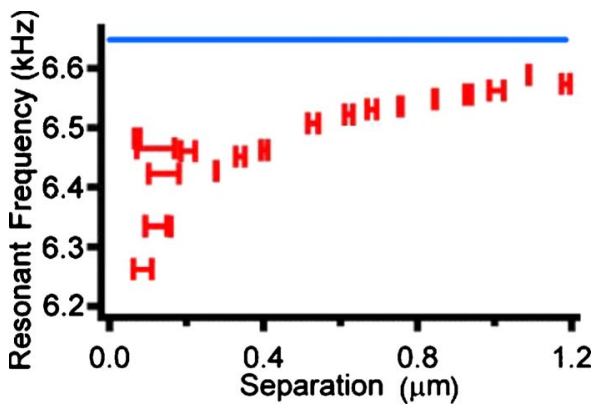

FIG. 5. (Color online) Resonant frequency measured from the $E_{\mathrm{SD}}$ fit, as a function of separation (red bars). The range of the error bars represent the separation measurement before and after the dwell. The resonant frequency measured at large separation for a period of $200 \mathrm{~s}$ is also shown (blue). Far field resonant frequency $=6.648 \mathrm{kHz}$, Spring constant $=0.0548 \mathrm{~N} / \mathrm{m}$. Same cantilever from Fig. 2.
ORC8-D cantilevers used here, the damping asymptotes to the bulk damping value at about $20 \mu \mathrm{m}$ separation. That is, at a separation of $30 \mu \mathrm{m}$ (the separation of the cantilever when an attached sphere in contact with the plate) the damping is approximately constant (see Fig. 4). So, in our experiments with attached spheres, we treated the damping from the cantilever as a constant, which was obtained at large separations.

When using triangular cantilevers, wider rectangular cantilevers (with a larger cantilever area) or smaller diameter spheres, the effect of cantilever proximity to the plate cannot be neglected. To correct for the effect of proximity to the plate, we take a second cantilever of the same dimensions, but without a sphere attached, and drive it toward the plate from a distance of $2 R$ while measuring the lubrication force by the thermal noise method. This gives us the form of the cantilever damping as a function of separation. We take this damping vs separation plot and scale it by the ratio of the bulk damping of the experimental cantilever (with a sphere) divided by the reference cantilever (with no sphere). We then have an estimate of the lubrication force damping from the experimental cantilever alone at a separation value of $2 R$.

Our fit of Eq. (7) to the $E_{\mathrm{SD}}$ S (Fig. 3) also returns the resonant frequency of the cantilever. The measured resonant frequency is shown as a function of separation in Fig. 5 (red bars). Figure 5 also shows the resonant frequency as measured by the Asylum Research software when no surface is present (blue line).

At smaller separations, there is greater scatter in the measured resonant frequency. At these smaller separations, there is greater damping on the cantilever, which causes a broader resonance peak and therefore greater error in fitting to Eq. (7). For example, compare the two curves shown in Fig. 3. Clearly the resonance peak at $1.091 \mu \mathrm{m}$ separation (quality factor $=19.86$ ) is more sharply defined and therefore easier to identify than the peak at $71 \mathrm{~nm}$ (quality factor $=4.88$ ).

We now return to our principal aim, which is to compare the sphere damping measured by the thermal method to the theoretical lubrication force, described by Eq. (4). First, Eq. (4) is recast so that it is linear in separation

$$
-\frac{1}{F} \frac{d s}{d t}=\frac{1}{D}=\frac{1}{6 \pi \eta R^{2}}\left(s+\delta_{\text {total }}\right) .
$$

In this form, the inverse damping coefficient vs separation is linear and the combined slip length for both surfaces can be determined directly from minus the $1 / D=0$ intercept of a linear fit, and the viscosity is proportional to the slope. The plot $1 / D$ vs separation, $s$, does indeed yield a straight line (Fig. 6, red bars), in agreement with Eq. (4). We also note that there is a finite intercept, demonstrating that there is a finite slip length, as expected for a gas.

An alternative theoretical model has been presented by Vinogradova for a sphere approaching a plate. This model treats the slip length as a shear independent constant [Eq. (3)] but makes a higher order approximation by considering the curvature of the sphere as it approaches the flat surface. For two identical surfaces with the same slip length [31]

$$
-\frac{1}{F} \frac{d s}{d t}=\frac{1}{6 \pi \eta R^{2} \Psi} s,
$$




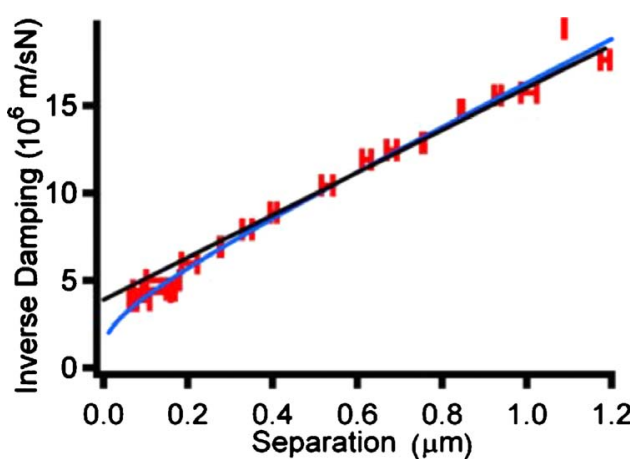

FIG. 6. (Color online) Inverse damping as a function of separation obtained by analysis of the $E_{\mathrm{SD}}$ in the dwell thermal noise method (red bars). A linear fit to the data between $200 \mathrm{~nm}$ and $1.2 \mu \mathrm{m}$ is shown in black and extrapolated to zero separation. A fit of the Vinogradova theory over the same range is shown in blue. The linear fit returns a slip length of $320 \mathrm{~nm}$ and a viscosity of $0.0186 \mathrm{mPa}$ s (black line). The Vinogradova fit returns a slip length of $392 \mathrm{~nm}$, when the viscosity is fixed at the same value as the linear fit, $0.0186 \mathrm{mPa}$ s (blue line). The range of the error bars represents the separation measurement before and after the dwell. The cantilever is the same as in Fig. 2.

$$
\Psi=\frac{s}{1.5 \delta_{\text {total }}}\left[\left(1+\frac{s}{3 \delta_{\text {total }}}\right) \ln \left(1+\frac{3 \delta_{\text {total }}}{s}\right)-1\right] .
$$

At large separation where this alternative formulation is strictly valid, this equation gives the same result as Eq. (8). Interestingly, we find that the Vinogradova solution fits the experimental data with greater precision (see Fig. 6) at smaller separations, i.e., in the transition regime, even though the Vinogradova solution is only strictly valid in the slip regime, i.e., at small $\mathrm{Kn}$ corresponding to large separation.

As the first-order slip boundary condition is only valid for small Knudsen number, Eqs. (8) and (9) can only be used in a regime where the mean-free path can be considered small relative to the separation. We therefore do not fit Eqs. (8) and (9) to data below $200 \mathrm{~nm}$. In the linear fit [Eq. (8)] to the data in Fig. 6 the slip length is obtained from the separationaxis intercept and the viscosity from the gradient of the line. Therefore a systematic error (for example, in the spring constant or sphere radius) has no effect on the measured slip length. In the Vinogradova solution the gradient and intercept are not independent. So although the Vinogradova and linear fits agree with high precision between $200 \mathrm{~nm}$ and $1.2 \mu \mathrm{m}$, the measured slip lengths and viscosities are different.

A sample of the data from the continuous thermal noise method is shown in Fig. 7 (as red circles) at a piezo displacement approach rate of $25.2 \mathrm{~nm} / \mathrm{s}$. Overlaid with this data is a set of data from the dc force curve, driven at an approach rate of $2.19 \mathrm{~mm} / \mathrm{s}$. The agreement of these two independent measurements of the lubrication force damping gives confidence that the analysis has been performed correctly. A linear fit between 200 and $600 \mathrm{~nm}$ returns a measured total slip length of $270 \mathrm{~nm}$. The measured air viscosity from the gradient of the linear fit is $0.017 \mathrm{mPa} \mathrm{s}$, compared to a bulk value of $0.02 \mathrm{mPas}$. The fit is not performed for $s$

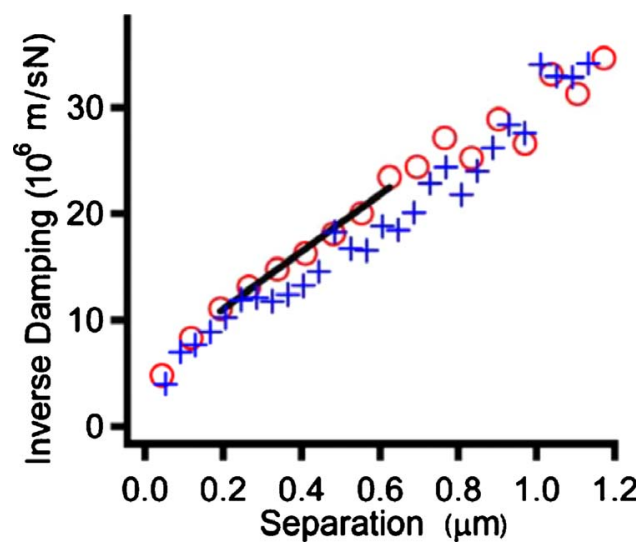

FIG. 7. (Color online) Inverse damping coefficient as a function of separation obtained by analysis of the $E_{\mathrm{SD}}$ in a slow $(25.2 \mathrm{~nm} / \mathrm{s})$ approach (red circles). A linear fit to the data between 200 and 600 $\mathrm{nm}$ is shown is black and returns a slip length of $270 \mathrm{~nm}$ and a viscosity of $0.017 \mathrm{mPa}$. The damping coefficient can also be obtained from the deflection of the cantilever in a separate high-speed (2.19 $\mathrm{mm} / \mathrm{s})$ approach (blue crosses). Gold coated silicon nitride cantilever Veeco MSCT-D, sphere radius $=11.2 \mu \mathrm{m}$, and cantilever spring constant $($ rectangle $)=0.031 \mathrm{~N} / \mathrm{m}$.

$>600 \mathrm{~nm}$ because in this run, the cantilever drag is a large component of the total damping and above $600 \mathrm{~nm}$ it becomes comparable to the lubrication force. Therefore the data above $600 \mathrm{~nm}$ is less reliable.

Table I is a summary of the measured combined slip length, accommodation coefficient and viscosity for all experiments, using a linear fit to the data. We list the combined slip length (from the two surfaces) because this is what we measure in our experiment. Although the chemistry on the sphere and plate are the same, the surface roughness is not, so the slip length may be different on the two solids. To determine the accommodation coefficient, we assume that

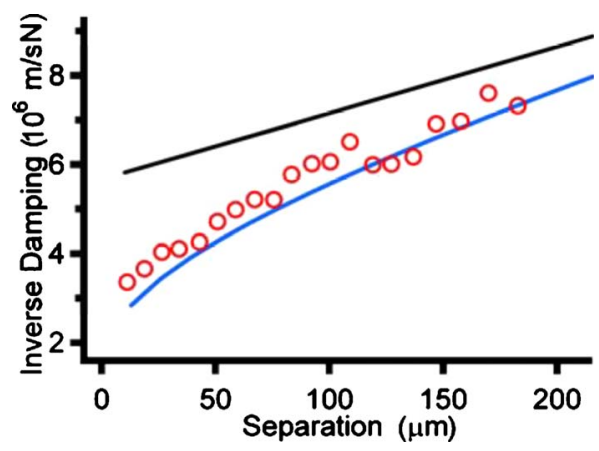

FIG. 8. (Color online) Measured inverse damping coefficient as a function of separation for a short-range continuous-run approach between 0 and $200 \mathrm{~nm}$. The extrapolation of the linear fit in the range of 200-1200 nm to Eq. (4) is shown in black and the extrapolation of the fit of the same data to the Vinogradova equation [Eq. (9)] is shown in light blue. For the linear fit, the measured combined slip length was $320 \mathrm{~nm}$ and the viscosity was $0.0186 \mathrm{mPa}$. For the Vinogradova equation, the viscosity was held to the same value as the linear fit (0.0186 $\mathrm{mPa} \mathrm{s})$, and the fitted combined slip length was $392 \mathrm{~nm}$. Sphere radius $=12.7 \mu \mathrm{m}$ and cantilever spring constant $($ triangle $)=0.019 \mathrm{~N} / \mathrm{m}$. 
TABLE I. The measured total slip length (combined from both surfaces) and viscosity for eight different experiments measured from the linear fits, using the dwell method and the continuous method. Two approach runs are tabulated for each "continuous" experiment. The accommodation coefficient $\sigma$ is calculated by assuming the slip length is the same on the sphere and the plate, and that the mean free path of air in $66 \mathrm{~nm}$.

\begin{tabular}{|c|c|c|c|c|c|}
\hline Experiment No. & Cantilever geometry & $\begin{array}{l}\text { Combined slip length } \\
\text { (nm) }\end{array}$ & $\sigma$ & $\begin{array}{l}\text { Viscosity } \\
(\mathrm{mPa} \mathrm{s})\end{array}$ & $\begin{array}{l}\text { Sphere radius } \\
(\mu \mathrm{m})\end{array}$ \\
\hline \multicolumn{6}{|c|}{ Dwell Thermal Noise Method } \\
\hline 1 & Rectangle & 320 & 0.62 & 0.0186 & 15.3 \\
\hline 2 & Rectangle & 274 & 0.69 & 0.0201 & 15.9 \\
\hline Average $1-2$ & & 297 & & & \\
\hline \multicolumn{6}{|c|}{ Continuous Thermal Noise Method } \\
\hline 3.1 & Triangle & 426 & 0.50 & 0.0162 & 11.7 \\
\hline 3.2 & & 262 & 0.71 & 0.0146 & \\
\hline 4.1 & Triangle & 326 & 0.61 & 0.0156 & 12.7 \\
\hline 4.2 & & 402 & 0.52 & 0.0170 & \\
\hline 5.1 & Triangle & 250 & 0.73 & 0.0172 & 12.7 \\
\hline 5.2 & & 295 & 0.66 & 0.0186 & \\
\hline 6.1 & Triangle & 95 & 1.22 & 0.0137 & 12.7 \\
\hline 6.2 & & 205 & 0.83 & 0.0161 & \\
\hline 7.1 & Rectangle & 72 & 1.35 & 0.0206 & 11.4 \\
\hline 7.2 & & 206 & 0.83 & 0.0259 & \\
\hline 8.1 & Rectangle & 223 & 0.79 & 0.0254 & 11.2 \\
\hline 8.2 & & 239 & 0.76 & 0.0269 & \\
\hline Average 3-8 & & 250 & & & \\
\hline Average $1-8$ & & 257 & 0.77 & 0.0190 & \\
\hline Standard deviatic & -8 & 99.0 & 0.24 & 0.0043 & \\
\hline
\end{tabular}

the slip length on each surface is equal and use a mean free path of $66 \mathrm{~nm}$ for air.

For comparison, the average across 12 repeat runs by the dc method from experiment 6 returns a slip length of $252 \pm 85 \mathrm{~nm}$ standard deviation and a measured viscosity of $0.021 \pm 0.002 \mathrm{mPa}$. We note that the viscosity of air is relatively insensitive to variations in temperature and humidity and is independent of pressure; it is expected to be $0.0186 \mathrm{mPas}$ for the present case. As such, the observed maximum deviations (approximately $\pm 30 \%$ ) in the measured viscosity in Table I are due to uncertainties and systematic errors in the fits and measured parameters; this is consistent with the expected uncertainties in cantilever calibration, sphere dimensions and fitting of the damping curves.

We can easily measure the damping coefficient at separations below $200 \mathrm{~nm}$, as shown in Fig. 8. These data do not fit to the extrapolation of Eq. (8) from the data in the range of 200-600 $\mathrm{nm}$. The data generally follow the extrapolation of Eq. (9) but not with as high precision as the data between 200 and $600 \mathrm{~nm}$. This is not unexpected, as the range is outside the validity of the slip-flow regime; i.e., the Knudsen number is approaching 1 . To the best of our knowledge, there is no complete theory to compare to the data in this range.

\section{DISCUSSION}

\section{A. Validation of the thermal noise method}

We measure the slip length by two methods: the thermal noise method and high-speed dc drive. The velocity divided by the force from the high-speed dc measurement is also plotted against separation in Fig. 7 (blue crosses). These two data sets are from separate experiments at different approach speeds with completely different analysis. To illustrate this, consider that the average approach speed in the dc measurement is five orders of magnitude greater than the approach speed in the thermal method. The velocity of the tip undergoing thermal motion is on the order of $1-10 \mu \mathrm{m} / \mathrm{s}$, which is two to three orders of magnitude below the piezo displacement drive speed in the dc method. It is important to stress that although the analysis of the damping by the thermal method and the dc method are completely independent, they have a high level of agreement (Fig. 7). This agreement provides strong evidence that the initial assumptions of Eq. (6) and its implementation are valid.

Over the range of $0-200 \mathrm{~nm}$ of separation, there is a systematic drop in the measured resonant frequency of the cantilever. This is not due to noise in the $E_{\mathrm{SD}}$ fit but is due to surface forces present in the experiment which alter the ef- 


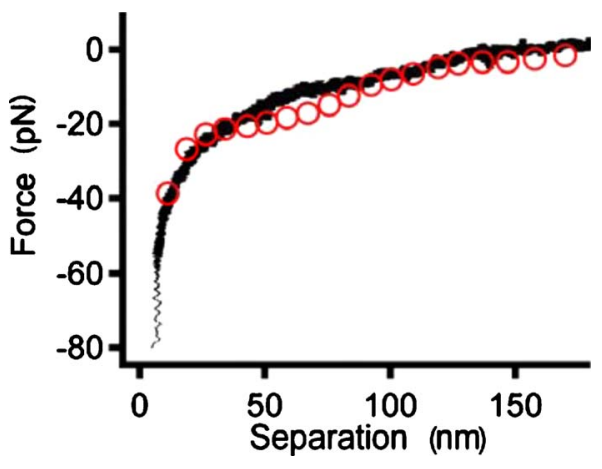

FIG. 9. (Color online) Quasistatic surface force as a function of separation during a slow approach, calculated in two ways: (1) directly from the deflection (black) and (2) from the shift in resonant frequency of the cantilever, using Eq. (10) (red circles). The agreement between these independent measurements of the surface force gives confidence that the analysis has been performed correctly. Same cantilever from Fig. 7.

fective spring constant of the cantilever. Ducker et al. [25] previously described how this shift in resonant frequency can be used to determine the surface force $F_{\text {surface }}(z)$

$$
F_{\text {surface }}(z)=\int_{0}^{z} k\left(1-\frac{f_{0}(z)^{2}}{f_{0}(\infty)^{2}}\right) d z
$$

where $f_{0}(z)$ represents the resonant frequency at a given equilibrium separation and $f_{0}(\infty)$ is the resonant frequency with no surface present. The surface force calculated from the resonance shift is shown in Fig. 9 (red circles). We also took the same raw deflection data and smoothed it to remove the thermal oscillations. Because the data are from a slow run, the only terms left in Eq. (5) are the spring force and the surface forces, so the surface force can be determined directly from the average spring deflection and this is shown in Fig. 5 (black points). The high level of agreement between these two independent methods of analyzing the data again provides strong evidence that the initial equation of motion and the analysis methodology are correct.

A further validation of the initial assumptions is that the measured gradient of the inverse damping coefficient yields a constant viscosity that agrees with the bulk viscosity of air within error. We measure an average air viscosity of $0.019 \pm 0.005 \mathrm{mPa} \mathrm{s}$, which compares with a known bulk value of $0.0186 \mathrm{mPa}$. The error in measured viscosity does not include the systematic error in the spring constant.

Why is there such good agreement between the damping coefficients measured by the dc and thermal methods (4-8 $\mathrm{kHz})$ ? In general the damping coefficient of a resonator in a viscous fluid is frequency dependent. The thickness of the viscous boundary layer around the oscillator can change as a function of frequency which changes the amount of damping.

To understand the damping on the oscillating sphere, we need to examine the hydrodynamics of the flow. A critical parameter to the flow around the oscillating sphere is the boundary layer thickness $(b)$

$$
b=\sqrt{\frac{\eta}{\pi f \rho}},
$$

where $\eta$ is the fluid viscosity, $f$ is the frequency of the cantilever, and $\rho$ is the fluid density. In air, for an oscillation frequency of $8 \mathrm{kHz}$, the boundary layer thickness is $28 \mu \mathrm{m}$. So the boundary layer is an order of magnitude greater than the separations that we measure. This means that the squeeze film flow between the sphere and the surface, which dominates the measured hydrodynamic force, is in the low inertia regime. As such, the hydrodynamic force will be frequency independent. We also note that the flow is in the linear Stokes regime, so the drag force is linearly proportional to the velocity and we expect the damping coefficient to be constant.

\section{B. Slip length as a parameter}

In general, the slip length is not the ideal parameter to use to characterize the analysis of slip-flow measurements that we perform using AFM at atmospheric or lower pressure with the linear model [Eq. (4)]. The difficulty is that the slip-flow regime is applicable only at separations significantly greater than the mean free path. Thus, the data is collected at separations greater than twice the mean free path $\mathrm{nm}$, and then extrapolated to a negative separation of about twice the mean free path to obtain the slip length. For the work here, this extrapolation was from $+200 \mathrm{~nm}$ to approximately $-200 \mathrm{~nm}$. This long extrapolation is the principal source of error in our measurements of the slip length or accommodation coefficient. As an alternative, an extrapolation to the damping coefficient at zero separation would be a parameter with a smaller associated error.

\section{Comparison between the dc and thermal methods}

In the thermal method, the damping coefficient and the resonant frequency are independent parameters in the fit of the data to the equation of motion, so the damping coefficient is independent of the magnitude of surface forces. As long as the gradient of the surface force can be considered constant (or negligible) over the amplitude of the cantilever oscillation, surface forces do not affect the thermal method. We often observe an attractive, low gradient equilibrium force that arises from the build up of electrostatic charge on the plate. (This force is consistent with the formation of induced dipoles on the sphere and plate.) We find that the slip length and viscosity measured by the thermal method are the same regardless of the presence or absence of this force, even when the electrostatic force has the same magnitude as the lubrication force. In contrast, the dc method measures the sum of the forces. So the electrostatic force must be subtracted from the total force in the dc method, which introduces a large error when the static and lubrication forces are similar in magnitude. The independence of the static and dynamic contributions of the force is a major advantage of the thermal noise method over the dc method.

An additional advantage of the thermal noise method is that the approach speeds are very low, so there is no ringing of the cantilever [7]. The disadvantages of the thermal noise 
method are (1) it relies on the correct equation of motion (which we have validated) (2) the continuous method is very slow, so thermal drift can be a problem and (3) it requires capture of large amounts of data. For example, each data set in the continuous method required 100 megabytes of storage.

\section{Comparison between the continuous and dwell thermal noise methods}

The presumed advantage of the dwell method is that the increased time at each separation allows better measurement of the $E_{\mathrm{SD}}$. In our measurements, the period of the dwell at each separation was $20 \mathrm{~s}$, whereas in the continuous measurement, the period over which we binned data was 2 s. Comparison between the data in Figs. 6 and 7 shows that there is less scatter in the damping for the dwell method, as expected. The data in Table I tell much the same story. The small number of data points for the dwell method prevents a rigorous comparison, but both dwell measurements produced a viscosity and slip length near the average whereas there was a large scatter in the continuous method. The errors in separation due to drift in the dwell method and continuous method are similar.

\section{E. Comparison between the thermal noise method and the driven oscillator method}

Maali and Bhushan [14] measured lubrication forces by driving the cantilever at a single frequency and monitoring the phase and amplitude response [19]. An advantage of the driven cantilever is that only the amplitude and phase response at a single drive frequency need to be measured, so the measurement is much faster. An advantage of the thermal noise method is that it is offers the minimum perturbation: for the same cantilever, the amplitude of vibration will be larger for the driven method, resulting in a loss of distance resolution. Also, the thermal method is an equilibrium method, so measures the equilibrium properties of the thin film, whereas the driven method has a flux of energy from the cantilever, which may heat the thin film between the probe and the plate. By experiment, we find that the area under the $E_{\mathrm{SD}}$ is independent of separation, so we do not resolve any change in the temperature of the cantilever during the approach. The thermal method also measures the entire resonance peak, which can be used to validate the equations of motion. However, it is possible to drive the cantilever over a range of frequencies to collect the entire spectrum [25]. Finally, the driven method relies on the measurement of phase, and small phase offsets can introduce errors in the analysis.

\section{F. Comparison to measurements of accommodation coefficients in similar systems}

We measure $\sigma=0.77 \pm 0.24$ for TMCS-coated silica. Other studies have found similar values for uncoated silicon. In a microchannel experiment, Veijola et al. measured a TMAC of $0.621-0.661$ in air against silicon with a surface roughness of $1 \mathrm{~nm}$ [32]. In similar microchannel experiments, Jang and Werely measured a TMAC of 0.85 against silicon with a surface roughness of $6.4 \mathrm{~nm}$ in air [33]. Huang et al. measured a larger TMAC of 0.90 in air against a glass surface, with a higher surface roughness $(70 \mathrm{~nm})$ [34].

\section{CONCLUSION}

We have demonstrated both a thermal noise method and a dc method for measuring lubrication forces in air. The data for both lubrication measurements show the expected functional form for damping in the slip-flow regime. We have measured a combined slip length of $250 \mathrm{~nm}$ for two hydrophobically coated glass solids, which corresponds to an accommodation coefficient, $\sigma=0.75$. We have also measured the lubrication force in the transition and free molecular flow regimes $(s<200 \mathrm{~nm})$. The data in this regime has a similar form to the Vinogradova equation. In addition, we show that the thermal measurement can be used to measure the quasistatic surface force in agreement with the dc method.

\section{ACKNOWLEDGMENTS}

This work was funded by both the Australian Research Council under Grant No. DP0664051 and the National Science Foundation under Grant No. CBET-0828163.
[1] Y. Sone, Kinetic Theory and Fluid Dynamics (Birkhauser, Boston, MA, 2002).

[2] G. Karniadakis, A. Beskok, and N. Aluru, Microflows: Fundamentals and Simulations (Springer, New York, 2002).

[3] C. R. Lilley and J. E. Sader, Proc. R. Soc. London, Ser. A 464, 2015 (2008).

[4] E. Lauga, M. P. Brenner, and H. A. Stone, in Handbook of Experimental Fluid Dynamics, edited by J. Foss, C. Tropea, and A. Yarin (Springer, New York, 2008), p. 17.

[5] C. Neto et al., Rep. Prog. Phys. 68, 2859 (2005).

[6] C. D. F. Honig and W. A. Ducker, Phys. Rev. Lett. 98, 028305 (2007).

[7] C. D. F. Honig and W. A. Ducker, J. Phys. Chem. C 111,
16300 (2007).

[8] C. D. F. Honig and W. A. Ducker, J. Phys. Chem. C 112, 17324 (2008).

[9] C. T. Rettner, IEEE Trans. Magn. 34, 2387 (1998).

[10] E. B. Arkilic, K. S. Breuer, and M. A. Schmidt, J. Fluid Mech. 437, 29 (2001).

[11] P. Tekasakul et al., J. Vac. Sci. Technol. A 14, 2946 (1996).

[12] A. Agrawal and S. V. Prabhu, J. Vac. Sci. Technol. A 26, 634 (2008).

[13] B.-Y. Cao et al., Int. J. Mol. Sci. 10, 4638 (2009).

[14] A. Maali and B. Bhushan, Phys. Rev. E 78, 027302 (2008).

[15] W. A. Ducker, T. J. Senden, and R. M. Pashley, Nature (London) 353, 239 (1991). 
[16] W. A. Ducker, T. J. Senden, and R. M. Pashley, Langmuir 8, 1831 (1992).

[17] H. J. Butt, Biophys. J. 60, 1438 (1991).

[18] S. Alexander et al., J. Appl. Phys. 65, 164 (1989).

[19] A. Roters and D. Johannsmann, J. Phys.: Condens. Matter 8, 7561 (1996).

[20] W. A. Ducker and R. F. Cook, Appl. Phys. Lett. 56, 2408 (1990).

[21] H. Brenner, Chem. Eng. Sci. 16, 242 (1961).

[22] J. L. Hutter and J. Bechhoefer, Rev. Sci. Instrum. 64, 1868 (1993).

[23] J. E. Sader et al., Rev. Sci. Instrum. 66, 3789 (1995).

[24] J. E. Sader, J. W. M. Chon, and P. Mulvaney, Rev. Sci. Instrum. 70, 3967 (1999).

[25] W. A. Ducker, R. F. Cook, and D. R. Clarke, J. Appl. Phys. 67,
4045 (1990).

[26] J. E. Sader, J. Appl. Phys. 84, 64 (1998).

[27] J. W. M. Chon, P. Mulvaney, and J. E. Sader, J. Appl. Phys. 87, 3978 (2000).

[28] C. P. Green and J. E. Sader, J. Appl. Phys. 98, 114913 (2005).

[29] A. P. French, Vibrations and Waves (Norton, New York, 1971).

[30] J. E. Sader et al., J. Appl. Phys. 97, 124903 (2005).

[31] O. I. Vinogradova, Int. J. Min. Process. 56, 31 (1999).

[32] T. Veijola, H. Kuisma, and J. Lahdenpera, Sens. Actuators, A, 66, 83 (1998).

[33] J. Jang and S. T. Wereley, J. Micromech. Microeng. 16, 493 (2006).

[34] C. Huang, J. W. Gregory, and J. P. Sullivan, J. Microelectromech. Syst. 16, 777 (2007). 